\title{
Fields of surreal numbers and exponentiation
}

\author{
by \\ Lou van den Dries (Urbana, IL) and \\ Philip Ehrlich (Athens, OH)
}

\begin{abstract}
We show that Conway's field of surreal numbers with its natural exponential function has the same elementary properties as the exponential field of real numbers. We obtain ordinal bounds on the length of products, reciprocals, exponentials and logarithms of surreal numbers in terms of the lengths of their inputs. It follows that the set of surreal numbers of length less than a given ordinal is a subfield of the field of all surreal numbers if and only if this ordinal is an $\varepsilon$-number. In that case, this field is even closed under surreal exponentiation, and is an elementary extension of the real exponential field.
\end{abstract}

Introduction. Conway [1] introduced the ordered field No of surreal numbers, which extends the field $\mathbb{R}$ of real numbers. (See Section 1 for a brief account of No.) Gonshor ([6], Ch. 10) followed suggestions by Kruskal and defined an exponential function exp $: \mathbf{N o} \rightarrow \mathbf{N o}$ such that $\exp (x)=e^{x}$ for $x \in \mathbb{R}$. In Section 2 below we show that No with exp is an elementary extension of the real exponential field: elementary statements true in the real exponential field remain true in the exponential field of surreal numbers. (See Wilkie [10], and Macintyre and Wilkie [9] for information on the elementary theory of the real exponential field.)

This result relating real and surreal exponentiation was also noticed by A. Macintyre, by M. H. Mourgues, and by J. Lurie [8], and answers a question of the first author in [2], p. 8. The proof below consists in equipping No with even further structure, by extending the restricted analytic functions from the real field to No, and verifying that the axioms in [3] for the model-complete theory $T_{\text {an,exp }}$ are satisfied by the thus expanded No.

The original content of the paper lies almost entirely in Sections 3-5, which contain the following results. Let $\operatorname{No}(\lambda)$ be the set of surreals of

2000 Mathematics Subject Classification: Primary 03C64, 03C65, 03H05, 12J15, 20F60; Secondary 04A10, 06F.

Key words and phrases: surreal numbers, exponential fields.

Research supported by the National Science Foundation. 
length less than the ordinal $\lambda$ (see below for "length"). Then $\mathbb{R} \subseteq \mathbf{N o}(\lambda)$ for $\lambda>\omega$. The ordinals $\lambda$ such that $\operatorname{No}(\lambda)$ is closed under the various operations of No can be characterized as follows:

(1) $\mathbf{N o}(\lambda)$ is an additive subgroup of No iff $\lambda=\omega^{\alpha}$ for some ordinal $\alpha$.

(2) $\operatorname{No}(\lambda)$ is a subring of No iff $\lambda=\omega^{\omega^{\alpha}}$ for some ordinal $\alpha$.

(3) $\mathbf{N o}(\lambda)$ is a subfield of No iff $\omega^{\lambda}=\lambda$. Moreover, if $\mathbf{N o}(\lambda)$ is a subfield of No, then it is also closed under exp, and is an elementary substructure of the exponential field No.

Here we used the customary notation for ordinal exponentiation with base $\omega$. Ordinals $\lambda$ such that $\omega^{\lambda}=\lambda$ are called $\varepsilon$-numbers. The smallest $\varepsilon$-number is the countable ordinal

$$
\varepsilon_{0}:=\sup \left\{\omega, \omega^{\omega}, \omega^{\omega^{\omega}}, \ldots\right\} .
$$

These results will be deduced from bounds on the length of $P\left(a_{1}, \ldots, a_{n}\right)$ in terms of the lengths of surreals $a_{1}, \ldots, a_{n}$, for various surreal operations $P$. The bulk of the work goes into establishing these bounds, which we consider of independent interest.

1. Background on surreal numbers. We define here No and point out some of its remarkable and distinctive features. We also take the opportunity to fix notations and terminology, which vary slightly across the literature $[1,4,6]$.

As in [6] a surreal number is by definition a function $a: \lambda \rightarrow\{-,+\}$ where $\lambda$ is an ordinal. For such $a$ we put $l(a):=\lambda$, the length of $a$ as in [6], which equals the tree-rank of $a$ in the canonical binary tree underlying No as treated in [4]. Thus the totality of surreal numbers, the "universe" of No, is not a set, but a proper class. To formalize this notion of "proper class" we use NBG (von Neumann-Bernays-Gödel set theory with Global Choice) as our underlying set theory (which is conservative over ZFC). Although truth is not in general definable in NBG for structures whose universe is a proper class, one can develop enough basic algebra and model theory in NBG to justify the considerations below. For details on these foundational matters we refer the reader to [5].

The class No carries a canonical linear ordering: $a<b$ iff $a$ is lexicographically less than $b$, where we set $a(\mu):=0$ for each surreal $a$ and ordinal $\mu \geq l(a)$ and linearly order $\{-, 0,+\}$ by $-<0<+$. As in [4] we define the canonical partial ordering $<_{s}$ on No by: $a<_{s} b$ (" $a$ is simpler than $b$ ") iff $a$ is a proper initial segment of $b$. A set $S \subseteq$ No is said to be initial if for all $x, y \in \mathbf{N o}, x<_{s} y \in S$ implies $x \in S$. (Thus $\mathbf{N o}(\lambda)$ is an initial subset of No for each ordinal $\lambda$.) 
KeY FACT. For any subsets $L$ and $R$ of No with $L<R$ there is a (unique) simplest $a \in$ No with $L<a<R$, notation: $a=\{L \mid R\}$. (NB: this would fail if we had allowed $L$ and $R$ to be subclasses, and also if No were replaced by any subset. Thus the distinction between sets and classes is essential in this context.)

Often we use expressions like $a=\left\{a^{L} \mid a^{R}\right\}$ to mean $a=\{L \mid R\}$, it being understood that $a^{L}$ ranges over $L$ and $a^{R}$ over $R$. For surreal $a$ we let $L_{s(a)}:=\left\{x \in\right.$ No $\left.: x<_{s} a, x<a\right\}$ and $R_{s(a)}:=\left\{x \in\right.$ No $\left.: x<_{s} a, x>a\right\}$; then $a=\left\{L_{s(a)} \mid R_{s(a)}\right\}$.

The key fact above allows us to introduce operations on No by recursion (similar to transfinite recursion and recursive definitions of functions on $\mathbb{N}$ ) and supports proofs by induction. For example, requiring translation invariance of $<$ for a binary operation of addition on No suggests that we define addition by the recursion $a+b=\left\{a^{L}+b, a+b^{L} \mid a^{R}+b, a+b^{R}\right\}$ with $a^{L}, b^{L}$, $a^{R}, b^{R}$ ranging over $L_{s(a)}, L_{s(b)}, R_{s(a)}$, and $R_{s(b)}$ respectively. Along these lines we are also led to the recursive definition of multiplication. Remarkably, this gives much more than we gambled for: No is an ordered real closed field with respect to $<$ and these operations of addition and multiplication. Moreover, there is a characterization of No up to unique isomorphism as an "ordered field with $<_{s}$ ", which is reminiscent of the familiar characterization of the ordered field of real numbers as the up to isomorphism unique complete ordered field. See [4] for more on this.

Ordinals are considered as surreal numbers by identifying each ordinal $\lambda$ with the surreal number of length $\lambda$ having only plus signs. The ordinals 0 and 1 are just the additive zero and multiplicative unit of the field No. The ordering, addition and multiplication of No restricted to the class $O n$ of ordinals coincide with the usual ordering of $O n$, and with the natural sum and natural product (see [7], pp. 80-81). We also have occasion to use the ordinal sum and ordinal product, writing these as $\alpha \dot{+} \beta$ and $\alpha \dot{\times} \beta$ to avoid confusion. (Throughout, $\alpha, \beta, \lambda, \mu, \nu$ are ordinals.)

The surreals of length $<\omega$ are exactly the dyadic rationals of the ordered field No. Throughout we identify the real field $\mathbb{R}$ with the unique initial subfield of No that is isomorphic to $\mathbb{R}$. Hence $l(r) \leq \omega$ for $r \in \mathbb{R}$. For more details on these identifications, see $[1,4,6]$.

Another striking surreal feature is that ordinal exponentiation with base $\omega$ extends to an operation $x \mapsto \omega^{x}:$ No $\rightarrow$ No in such a way that every surreal number can be written uniquely as a generalized power series in $\omega$ with real coefficients and surreal exponents. We make heavy use of this representation in what follows. More precisely, for any ordinal $\beta$, any strictly decreasing family $\left(y_{\alpha}\right)_{\alpha<\beta}$ of surreals, and any family $\left(r_{\alpha}\right)_{\alpha<\beta}$ of non-zero real numbers, the expression $\sum_{\alpha<\beta} \omega^{y_{\alpha}} \cdot r_{\alpha}$ denotes a surreal number $a$, and 
is called the normal form of $a$ in [1] and [6]. Following [4] we shall call it the Conway name of $a$. Each surreal number has a unique Conway name, and the Conway name of an ordinal is just its Cantor normal form (see [4], Theorems 22 and 23).

2. The elementary theory of No as exponential field. This section is primarily an extended observation. It involves describing No as a canonical directed union of copies of Hahn fields. This description is also used in later sections.

For any ordered abelian (additively written) group $\Gamma$ the ordered Hahn field $\mathbb{R}\left(\left(t^{\Gamma}\right)\right)$ is expanded in [3], p. 188 to an $L_{\text {an }}$-structure denoted there by $\mathbb{R}\left(\left(t^{\Gamma}\right)\right)_{\text {an }}$. If $\Gamma$ is divisible, then $\mathbb{R}\left(\left(t^{\Gamma}\right)\right)$ an is an elementary extension of $\mathbb{R}_{\text {an }}$, the field of reals with restricted analytic functions as defined in [3].

Here and later in this paper $\Gamma$ and other groups indicated by capital Greek letters are assumed to have as their universe a set, as opposed to a proper class.

For each additive subgroup $\Gamma$ of No we have a canonical embedding

$$
\sum_{\gamma \in \Gamma} s_{\gamma} t^{\gamma} \mapsto \sum_{\gamma \in \Gamma} s_{\gamma} \omega^{-\gamma}: \mathbb{R}\left(\left(t^{\Gamma}\right)\right) \rightarrow \text { No }
$$

of ordered fields. Here the infinite sum on the right denotes the surreal number with Conway name $\sum_{\alpha<\beta} \omega^{y_{\alpha}} \cdot r_{\alpha}$ where $\left(y_{\alpha}\right)_{\alpha<\beta}$ is a strictly decreasing enumeration of the set $\left\{-\gamma: s_{\gamma} \neq 0\right\}$ and $r_{\alpha}=s_{\gamma}$ whenever $y_{\alpha}=-\gamma$.

Let $\tau:=\omega^{-1}$, so that the embedding above takes the form

$$
\sum_{\gamma \in \Gamma} s_{\gamma} t^{\gamma} \mapsto \sum_{\gamma \in \Gamma} s_{\gamma} \tau^{\gamma}
$$

We denote the image of this embedding by $\mathbb{R}\left(\left(\tau^{\Gamma}\right)\right)$.

For use in later sections we transfer the notion of "support" from power series to surreal numbers. The support of a surreal number $a=\sum_{\gamma \in \Gamma} s_{\gamma} \tau^{\gamma} \in$ $\mathbb{R}\left(\left(\tau^{\Gamma}\right)\right)$ is the well-ordered subset $\operatorname{supp}(a):=\left\{\gamma \in \Gamma: s_{\gamma} \neq 0\right\}$ of $\Gamma$.

We expand the ordered subfield $\mathbb{R}\left(\left(\tau^{\Gamma}\right)\right)$ of No to an $L_{\text {an }}$-structure $\mathbb{R}\left(\left(\tau^{\Gamma}\right)\right)_{\text {an }}$ in such a way that the above map is an isomorphism from $\mathbb{R}\left(\left(t^{\Gamma}\right)\right)_{\text {an }}$ onto $\mathbb{R}\left(\left(\tau^{\Gamma}\right)\right)_{\text {an }}$. It is routine to verify that if $\Delta$ is a second additive subgroup of No containing $\Gamma$, then

$$
\mathbb{R}\left(\left(\tau^{\Gamma}\right)\right)_{\text {an }} \subseteq \mathbb{R}\left(\left(\tau^{\Delta}\right)\right)_{\text {an }},
$$

where we use " $\subseteq$ " to indicate the substructure relation. Since the additive group of No is the directed union of its additive subgroups $\Gamma$ (with a set as their universe!), and each surreal number belongs to some $\mathbb{R}\left(\left(\tau^{\Gamma}\right)\right)$, it follows that the ordered field No can be expanded uniquely to an $L_{\text {an }}$-structure 
$\mathbf{N o}_{\text {an }}$ such that for each such $\Gamma$,

$$
\mathbb{R}\left(\left(\tau^{\Gamma}\right)\right)_{\text {an }} \subseteq \mathbf{N o}_{\text {an }} .
$$

For divisible $\Gamma$ we even have by [3] an elementary substructure:

$$
\mathbb{R}\left(\left(\tau^{\Gamma}\right)\right)_{\text {an }} \preceq \mathbf{N o}_{\text {an }} .
$$

In particular, $\mathbf{N o}_{\text {an }}$ is an elementary extension of $\mathbb{R}_{\mathrm{an}}$, and thus a model of its complete theory $T_{\text {an }}$. We shall refer to $\mathbf{N o}_{\text {an }}$ as "the field of surreal numbers with restricted analytic functions".

By [3] the elementary theory of the expansion of $\mathbb{R}_{\text {an }}$ by its exponential function $e^{x}$ is completely axiomatized by $T_{\text {an }}$, together with the "Ressayre axioms" which express that the exponential function is an order preserving isomorphism from the additive group of the underlying ordered field onto its positive multiplicative group such that

(1) the exponential of any $x>n^{2}$ is greater than $x^{n}$ (for $n=1,2, \ldots$ );

(2) the exponential of any $x$ with $-1 \leq x \leq 1$ equals $E(x)$ where $E$ is the function symbol of $L_{\text {an }}$ corresponding to the exponential power series $\sum(1 / n !) X^{n} \in \mathbb{R}[[X]]$.

Moreover, this elementary theory is model-complete and has other good properties such as o-minimality. (See [3] for these particular facts, and [2] for a general account of o-minimality.) It so happens that [6] (parts A and B of Ch. 10) establishes the "Ressayre axioms" for the exponential function on No, except those listed under (1) above (which are easy to verify). Thus we may conclude:

THEOREM 2.1. The field of surreal numbers equipped with restricted analytic functions and with exp is an elementary extension of the field of real numbers with restricted analytic functions and real exponentiation.

CoROLlary 2.2. The exponential field of surreal numbers is an elementary extension of the exponential field of real numbers.

Perhaps future research will clarify whether these results have natural analogues for other o-minimal expansions of the real field.

Another question comes up: when is a fragment $\operatorname{No}(\lambda)$ of No the underlying set of an elementary substructure of No with respect to the various natural operations on No? This leads to the material in the next sections, and fits in with Gonshor's suggestion ([6], p. 103) to pay special attention to the field of surreals of countable length, that is, to $\mathbf{N o}\left(\omega_{1}\right)$.

3. Bounds on lengths of sums and products. We consider here some inequalities for surreal numbers $a$ and $b$ :

$$
l(a+b) \leq l(a)+l(b) \text { and } l(a b) \leq l(a) l(b) .
$$


They are respectively a result [6], p. 95, and a conjecture [6], p. 96. We first record an easy consequence of the first inequality. Next we establish the second inequality for $a$ and $b$ of the form $\omega^{x} \cdot r$ with surreal $x$ and real $r$. (This case suffices for our purpose in the next section where we determine the subrings and subfields of No of the form $\operatorname{No}(\lambda)$.)

Corollary 3.1. $\mathbf{N o}(\lambda)$ is an additive subgroup of No if and only if $\lambda=\omega^{\alpha}$ for some $\alpha$.

Proof. Combine the first inequality with the well known fact that the ordinals $\omega^{\alpha}$ are exactly the ordinals $\lambda>0$ for which $\mu+\nu<\lambda$ whenever $\mu, \nu<\lambda$.

The ordinals of the form $\omega^{\alpha}$ are often said to be additively indecomposable but for the sake of brevity we shall just call them additive. The ordinals of the form $\lambda=\omega^{\omega^{\alpha}}$ are exactly the ordinals $\lambda>1$ such that $\mu \nu<\lambda$ whenever $\mu, \nu<\lambda$. They are frequently called multiplicatively indecomposable, and we shall refer to them as multiplicative.

We now begin a sequence of lemmas leading to a proof of $l(a b) \leq l(a) l(b)$ in the special case mentioned earlier.

Lemma 3.2. Let $a>0$ be a dyadic rational. Then $l(a)=l([a])+l(a-[a])$, where $[a]$ is the largest integer $\leq a$.

Proof. This is clear if $a \in \mathbb{N}$. Let $a \notin \mathbb{N}$, and write $a=[a]+i / 2^{m}$ where $0<m \in \mathbb{N}, 0<i<2^{m}$ and $i$ is odd. Then $l(a)=[a]+m+1=$ $l([a])+l\left(i / 2^{m}\right)$.

Lemma 3.3. Let $a, b \in \mathbb{R}$. Then $l(a b) \leq l(a) l(b)$.

Proof. Since $l(x)=l(-x) \leq \omega$ for all $x \in \mathbb{R}$, we may reduce to the case that $a, b>0$ and $l(a), l(b)<\omega$. So $a, b$ are dyadic rationals. Write $a=[a]+u$ and $b=[b]+v$. Then

$$
a b=[a][b]+[a] v+[b] u+u v .
$$

Note that $l(u v) \leq l(u) l(v)$ : this is clear for $u=0$ or $v=0$; otherwise $u=i / 2^{m}$ and $v=j / 2^{n}$ with $0<m, n \in \mathbb{N}$ and odd $i, j$ with $0<i<2^{m}$ and $0<j<2^{n}$, so $u v=i j / 2^{m+n}$, hence $l(u v)=m+n+1 \leq(m+1)+(n+1)=$ $l(u) l(v)$. In combination with the inequality $l(x+y) \leq l(x)+l(y)$ (which in particular implies $l(n x) \leq n l(x)$ for $n \in \mathbb{N})$ this gives

$$
\begin{aligned}
l(a b) & \leq l([a][b])+l([a] v)+l([b] u)+l(u v) \\
& \leq[a][b]+[a] l(v)+[b] l(u)+l(u) l(v) \\
& =([a]+l(u))([b]+l(v))=l(a) l(b),
\end{aligned}
$$

where the last equality uses the previous lemma. 
As in [6] we let $x^{+}$denote the ordinal number of plus signs in the surreal number $x$, that is, $x^{+}$is the ordinal obtained by deleting all minus signs in $x$. We also let $r^{\prime}$ be the real number obtained from a positive real number $r$ by deleting the initial plus sign of the sign sequence that constitutes $r$. With these notations we have:

Lemma 3.4. Let $x, y \in \mathbf{N o}$ and $0<r \in \mathbb{R}$. Then

(1) $(x+y)^{+} \leq x^{+}+y^{+}$,

(2) $l\left(\omega^{x}\right)=\omega^{x^{+}} \alpha$ for some ordinal $\alpha>0$ (hence $\left.l\left(\omega^{x}\right) \geq \omega^{x^{+}}\right)$,

(3) $l\left(\omega^{x} . r\right)=l\left(\omega^{x}\right) \dot{+}\left(\omega^{x^{+}} l\left(r^{\prime}\right)\right)$ (ordinal sum),

(4) if $r$ is a dyadic rational, then $l\left(\omega^{x} \cdot r\right)=l\left(\omega^{x}\right)+\omega^{x^{+}} l\left(r^{\prime}\right)$,

(5) if $r$ is not a dyadic rational, then $l\left(\omega^{x} . r\right)=l\left(\omega^{x}\right)+\omega^{x^{+}}(\omega-m)$ with $m \in \omega$ the coefficient of $\omega^{x^{+}}$in the Cantor normal form of $l\left(\omega^{x}\right)$.

Proof. Item (1) follows by an obvious inductive argument from $x+y=$ $\left\{x^{L}+y, x+y^{L} \mid x^{R}+y, x+y^{R}\right\}$ where $x^{L}, y^{L}, x^{R}, y^{R}$ range over elements of $L_{s(x)}, L_{s(y)}, R_{s(x)}$, and $R_{s(y)}$, respectively. Item (2) follows by induction on $l(x)$ using [6], Th. 5.11(a). Item (3) follows from [6], Th. 5.12(a). By (2), only powers $\omega^{\beta}$ with $\beta \geq x^{+}$can occur with a non-zero coefficient in the Cantor normal form of $l\left(\omega^{x}\right)$, and together with (3) this gives (4). In the same way we obtain (5).

Lemma 3.5. Let $x, y$ be surreals such that $l\left(\omega^{x} \omega^{y}\right) \leq l\left(\omega^{x}\right) l\left(\omega^{y}\right)$. Then

$$
l\left(\left(\omega^{x} \cdot r\right)\left(\omega^{y} . s\right)\right) \leq l\left(\omega^{x} \cdot r\right) l\left(\omega^{y} \cdot s\right)
$$

for all real numbers $r$ and $s$.

Proof. We may assume that $r$ and $s$ are positive real numbers. There are four cases.

CASE 1: $r$ and $s$ are dyadic rationals. Then Lemma 3.3 and parts (1), (2) and (4) of Lemma 3.4 lead to the following chain of equalities and inequalities:

$$
\begin{aligned}
l\left(\omega^{x} . r\right) l\left(\omega^{y} . s\right)= & \left(l\left(\omega^{x}\right)+\omega^{x^{+}} l\left(r^{\prime}\right)\right)\left(l\left(\omega^{y}\right)+\omega^{y^{+}} l\left(s^{\prime}\right)\right) \\
= & l\left(\omega^{x}\right) l\left(\omega^{y}\right)+l\left(\omega^{x}\right) \omega^{y^{+}} l\left(s^{\prime}\right) \\
& +\omega^{x^{+}} l\left(\omega^{y}\right) l\left(r^{\prime}\right)+\omega^{x^{+}} \omega^{y^{+}} l\left(r^{\prime}\right) l\left(s^{\prime}\right) \\
\geq & l\left(\omega^{x}\right) l\left(\omega^{y}\right)+\omega^{x^{+}} \omega^{y^{+}} l\left(s^{\prime}\right) \\
& +\omega^{x^{+}} \omega^{y^{+}} l\left(r^{\prime}\right)+\omega^{x^{+}} \omega^{y^{+}} l\left(r^{\prime}\right) l\left(s^{\prime}\right) \\
= & l\left(\omega^{x}\right) l\left(\omega^{y}\right)+\omega^{x^{+}} \omega^{y^{+}}\left(l\left(s^{\prime}\right)+l\left(r^{\prime}\right)+l\left(r^{\prime}\right) l\left(s^{\prime}\right)\right) \\
\geq & l\left(\omega^{x+y}\right)+\omega^{(x+y)^{+}} l\left((r s)^{\prime}\right)=l\left(\omega^{x+y} . r s\right)=l\left(\left(\omega^{x} . r\right)\left(\omega^{y} . s\right)\right) .
\end{aligned}
$$


Case 2: neither $r$ nor $s$ is a dyadic rational. Then by Lemma 3.4(5),

$$
l\left(\omega^{x} \cdot r\right)=l\left(\omega^{x}\right)+\omega^{x^{+}}(\omega-m) \text { and } l\left(\omega^{y} \cdot s\right)=l\left(\omega^{y}\right)+\omega^{y^{+}}(\omega-n)
$$

for certain $m, n \in \omega$. Similar inequalities to those in Case 1 lead to

$$
l\left(\omega^{x} \cdot r\right) l\left(\omega^{y} . s\right) \geq l\left(\omega^{x+y}\right)+\omega^{(x+y)^{+}}\left(\omega^{2}+p \omega+q\right)
$$

for certain $p, q \in \mathbb{Z}$. Now use the fact that $\omega^{2}+p \omega+q>\omega \geq l\left((r s)^{\prime}\right)$.

CASE 3: $r$ is a dyadic rational, $r \neq 1$, and $s$ is not a dyadic rational. Let $n$ be the coefficient of $\omega^{y^{+}}$in the Cantor normal form of $l\left(\omega^{y}\right)$, so $l\left(\omega^{y} . s\right)=l\left(\omega^{y}\right)+\omega^{y^{+}}(\omega-n)$ by Lemma 3.4(5). In the same way as before we obtain

$$
l\left(\omega^{x} \cdot r\right) l\left(\omega^{y} \cdot s\right) \geq l\left(\omega^{x+y}\right)+\omega^{(x+y)^{+}}\left(\omega-n+l\left(r^{\prime}\right)+l\left(r^{\prime}\right)(\omega-n)\right) .
$$

From $r \neq 1$ we get $l\left(r^{\prime}\right)>0$, hence $\omega-n+l\left(r^{\prime}\right)+l\left(r^{\prime}\right)(\omega-n)>\omega \geq l\left((r s)^{\prime}\right)$, and we are done by Lemma 3.4(3).

CASE $4: r=1$ and $s$ is not a dyadic rational. Let $s^{L}$ range over the surreals simpler and less than $s$, and $s^{R}$ over the surreals simpler and greater than $s$. So the $s^{L}$ 's are dyadic rationals cofinal in $\{s-\varepsilon: 0<\varepsilon \in \mathbb{R}\}$, and the $s^{R}$ 's are dyadic rationals coinitial in $\{s+\varepsilon: 0<\varepsilon \in \mathbb{R}\}$. Then by [6], Lemma 5.2, and [4], Th. 1(iii),

$$
\omega^{x} \omega^{y} . s=\omega^{x+y} . s=\left\{\omega^{x+y} \cdot s^{L} \mid \omega^{x+y} \cdot s^{R}\right\} .
$$

By Case 1 and Lemma 3.4 we have $l\left(\omega^{x+y} . s^{L}\right) \leq l\left(\omega^{x}\right) l\left(\omega^{y} \cdot s^{L}\right)<l\left(\omega^{x}\right) l\left(\omega^{y} . s\right)$, and similarly, $l\left(\omega^{x+y} . s^{R}\right)<l\left(\omega^{x}\right) l\left(\omega^{y} . s\right)$. Thus $l\left(\omega^{x+y} . s\right) \leq l\left(\omega^{x}\right) l\left(\omega^{y} . s\right)$, by [6], Th. 2.3.

Proposition 3.6. If $a=\omega^{x} . r$ and $b=\omega^{y} . s, x, y \in$ No, $r, s \in \mathbb{R}$, then $l(a b) \leq l(a) l(b)$.

Proof. By the previous lemma it suffices to prove $l\left(\omega^{x+y}\right) \leq l\left(\omega^{x}\right) l\left(\omega^{y}\right)$, and for this we proceed by induction on $l(x)$ and $l(y)$. The inequality clearly holds if $l(x)=0$ or $l(y)=0$. Let $l(x), l(y)>0$, and let $x^{L}, y^{L}, x^{R}, y^{R}$ and $n$ range over elements of $L_{s(x)}, L_{s(y)}, R_{s(x)}, R_{s(y)}$ and $\omega \backslash\{0\}$ respectively. Then $x+y=\left\{x^{L}+y, x+y^{L} \mid x^{R}+y, x+y^{R}\right\}$, hence by [4], Th. 12,

$$
\begin{aligned}
\omega^{x+y} & =\left\{0, \omega^{x^{L}+y} . n, \omega^{x+y^{L}} . n \mid \omega^{x^{R}+y} \cdot 2^{-n}, \omega^{x+y^{R}} \cdot 2^{-n}\right\} \\
& =\left\{0,\left(\omega^{x^{L}} . n\right) \omega^{y}, \omega^{x}\left(\omega^{y^{L}} . n\right) \mid\left(\omega^{x^{R}} \cdot 2^{-n}\right) \omega^{y}, \omega^{x}\left(\omega^{y^{R}} .2^{-n}\right)\right\} .
\end{aligned}
$$

Assume inductively that $l\left(\omega^{x^{L}} \omega^{y}\right) \leq l\left(\omega^{x^{L}}\right) l\left(\omega^{y}\right)$. Then by the last lemma we have $l\left(\left(\omega^{x^{L}} . n\right) \omega^{y}\right) \leq l\left(\omega^{x^{L}} . n\right) l\left(\omega^{y}\right)$. The first sign of $x$ after its initial segment $x^{L}$ is a plus, hence $\omega^{x^{L}} . n<_{s} \omega^{x}$ by [6], Th. 5.11, so $l\left(\omega^{x^{L}} . n\right)<$ $l\left(\omega^{x}\right)$, and thus $l\left(\left(\omega^{x^{L}} \cdot n\right) \omega^{y}\right)<l\left(\omega^{x}\right) l\left(\omega^{y}\right)$. Similar inductive assumptions 
imply that the other products listed in the representation of $\omega^{x+y}$ above all have length less than $l\left(\omega^{x}\right) l\left(\omega^{y}\right)$. Therefore $l\left(\omega^{x+y}\right) \leq l\left(\omega^{x}\right) l\left(\omega^{y}\right)$ by [6], Th. 2.3.

4. Bounds on lengths of infinite sums. In this section we prove that $\operatorname{No}(\lambda)$ is a subring of No if and only if $\lambda$ is multiplicative, and $\operatorname{No}(\lambda)$ is a subfield of No if and only if $\lambda$ is an $\varepsilon$-number. We also study the structure of these fields $\mathbf{N o}(\lambda)$. Several bounds on lengths that we need in this connection are improvements of bounds in [6], Ch. 6.

Lemma 4.1. Each surreal a satisfies $l(a) \leq l\left(\omega^{a}\right) \leq \omega^{l(a)}$.

Proof. Let $L:=L_{s(a)}$ and $R:=R_{s(a)}$, so that $a=\{L \mid R\}$. We assume inductively that the lemma holds for the surreals in $L \cup R$. From $\omega^{a}=\left\{0, \omega^{L} . n \mid \omega^{R} .2^{-n}\right\}$ (with $n$ ranging over $\mathbb{N}=\omega$ ) and [6], Th. 2.3, we obtain

$$
l\left(\omega^{a}\right) \leq \text { least ordinal } \lambda>0 \text { such that }
$$

$$
n \omega^{\alpha}<\lambda \text { for all } \alpha \in l(L \cup R), n \in \omega .
$$

For $\alpha \in l(L \cup R)$ we have $\alpha<l(a)$, and hence $n \omega^{\alpha}<\omega^{l(a)}$ for all $n$. Thus $l\left(\omega^{a}\right) \leq \omega^{l(a)}$. The other inequality follows from [4], Th. 12 .

REMARK. Both inequalities become equalities when $l(a)$ is an $\varepsilon$-number. By Lemma 4.8 below $l\left(\omega^{a}\right)<\omega^{l(a)}$ if $a=-\alpha$ for an ordinal $\alpha>1$ that is not an $\varepsilon$-number.

Lemma 4.2. Let the surreal number a have Conway name $\sum_{\alpha<\beta} \omega^{y_{\alpha}} \cdot r_{\alpha}$. Then

(1) $\beta \leq l(a)$,

(2) $l\left(\omega^{y_{\alpha}} \cdot r_{\alpha}\right) \leq l(a)$ for $\alpha<\beta$,

(3) if $\nu$ is such that $l\left(\omega^{y_{\alpha}} \cdot r_{\alpha}\right) \leq \nu$ for all $\alpha<\beta$, then $l(a) \leq \beta \nu$.

Proof. Inequality (1) is shown in [6], p. 63. The proof of [6], Lemma 6.3, gives inequality (2). For an upperbound $\nu$ as in (3) one shows by transfinite induction on ordinals $\mu \leq \beta$ that $l\left(\sum_{\alpha<\mu} \omega^{y_{\alpha}} \cdot r_{\alpha}\right) \leq \mu \nu$. (For a limit ordinal $\mu$ one can appeal to [6], Th. 2.3.)

Corollary 4.3. For any surreals $a$ and $b$ we have $l(a b) \leq \omega l(a)^{2} l(b)^{2}$.

Proof. Let $a, b$, and $a b$ have Conway names $\sum_{\alpha<\lambda} \omega^{x_{\alpha}} \cdot r_{\alpha}, \sum_{\beta<\mu} \omega^{y_{\beta}} \cdot s_{\beta}$, and $\sum_{\gamma<\nu} \omega^{z_{\gamma}} \cdot t_{\gamma}$. Since $\operatorname{supp}(a b) \subseteq \operatorname{supp}(a)+\operatorname{supp}(b)$, the order type of $\operatorname{supp}(a b)$ is at most $\lambda \mu$. Also, $\lambda \leq l(a)$ and $\mu \leq l(b)$ by part (1) of the last lemma. Hence $\nu \leq \lambda \mu \leq l(a) l(b)$. Moreover, each term $\omega^{z_{\gamma}} \cdot t_{\gamma}$ is a finite sum of products of the form $\left(\omega^{x_{\alpha}} \cdot r_{\alpha}\right)\left(\omega^{y_{\beta}} \cdot s_{\beta}\right)$. Each of these products has length at most $l\left(\omega^{x_{\alpha}} . r_{\alpha} \omega^{y_{\beta}} . s_{\beta}\right) \leq l(a) l(b)$, by Lemma 4.2(2) and Proposition 3.6. 
Thus $l\left(\omega^{z_{\gamma}} \cdot t_{\gamma}\right) \leq \omega l(a) l(b)$. Now use $\nu \leq l(a) l(b)$ and part (3) of the last lemma to reach the desired conclusion.

REMark. The proof shows that for $b$ of the form $\omega^{y} . s(y \in$ No, $s \in \mathbb{R})$ we can improve the inequality to $l(a b) \leq l(a)^{2} l(b)$.

COROllary 4.4. $\operatorname{No}(\lambda)$ is a subring of No if and only if $\lambda$ is multiplicative.

Next we have a quantitative version of a well known lemma of B. H. Neumann:

Lemma 4.5. Let $\Gamma$ be an ordered abelian group and $S$ a set of positive elements of $\Gamma$. Suppose $S$ is well ordered of order type $\mu$. Then the additive monoid $[S]$ generated by $S$ in $\Gamma$ is well ordered of order type at most $\omega^{\mu}$.

Proof. By an easy induction on $\mu$, using the fact that if $A, B \subseteq \Gamma$ are well ordered of order types $\alpha, \beta$, then $A+B$ is well ordered of order type at most $\alpha \beta$ (natural product of ordinals).

REMARK. Here is an analogue for semirings, which we shall not use: Let $K$ be an ordered field and let $S \subseteq K^{>0}$ be well ordered of order type $\mu$. Then the semiring generated by $S$ in $K$ (the smallest subset containing 0,1 and $S$ and closed under addition and multiplication) is well ordered of order type at most $\omega^{\omega^{\mu}}$.

Lemma 4.6. Let $\Gamma$ be an additive subgroup of No, $\lambda$ an $\varepsilon$-number, and put

$$
\mathbb{R}\left(\left(\tau^{\Gamma}\right)\right)_{\lambda}:=\left\{a \in \mathbb{R}\left(\left(\tau^{\Gamma}\right)\right): \operatorname{supp}(a) \text { has order type }<\lambda\right\} .
$$

Then $\mathbb{R}\left(\left(\tau^{\Gamma}\right)\right)_{\lambda}$ is a subfield of No, and is closed under the restricted analytic functions of No. If $\Gamma$ is also divisible, then $\mathbb{R}\left(\left(\tau^{\Gamma}\right)\right)_{\lambda}$ is real closed.

Proof. Let $a, b \in \mathbb{R}\left(\left(\tau^{\Gamma}\right)\right)_{\lambda}$, so $\operatorname{supp}(a)$ and $\operatorname{supp}(b)$ have order types $\alpha<\lambda$ and $\beta<\lambda$. Then $\operatorname{supp}(a+b)$ has order type at most $\alpha+\beta<\lambda$, and $\operatorname{supp}(a b)$ has order type at most $\alpha \beta<\lambda$. Suppose that $a \neq 0$. Write $a=r \omega^{y}(1-\varepsilon)$ with $r \in \mathbb{R} \backslash\{0\}$ and $\varepsilon$ infinitesimal. Clearly, the well ordered subset $\operatorname{supp}(\varepsilon)$ of $\Gamma^{>0}$ has order type at most $\alpha$. From $a^{-1}=r^{-1} \omega^{-y}\left(1+\varepsilon+\varepsilon^{2}+\ldots\right)$ it follows that

$$
\operatorname{supp}\left(a^{-1}\right)=-y+\operatorname{supp}\left(1+\varepsilon+\varepsilon^{2}+\ldots\right) \subseteq-y+[\operatorname{supp}(\varepsilon)]
$$

where [...] denotes the additive monoid generated by ..., as in the last lemma. This lemma then tells us that $\operatorname{supp}\left(a^{-1}\right)$ has order type at most $\omega^{\alpha}<\lambda$. Assume that in addition $a>0$ and $d$ is a positive integer. Then $r>0$ and $a^{1 / d}=r^{1 / d} \omega^{y / d} \sum_{n=0}^{\infty}(-1)^{n}\left(\begin{array}{c}1 / d \\ n\end{array}\right) \varepsilon^{n}$. A similar argument to that for $a^{-1}$ shows that the support of $a^{1 / d}$ has order type at most $\omega^{\alpha}<\lambda$, and thus $a^{1 / d} \in \mathbb{R}\left(\left(\tau^{\Gamma}\right)\right)_{\lambda}$ if $\Gamma$ is divisible. 
Finally, let $F\left(X_{1}, \ldots, X_{n}\right) \in \mathbb{R}\left[\left[X_{1}, \ldots, X_{n}\right]\right]$ be a formal power series in the indeterminates $X_{1}, \ldots, X_{n}$ with real coefficients. Let $\varepsilon_{1}, \ldots, \varepsilon_{n}$ be infinitesimals in $\mathbb{R}\left(\left(\tau^{\Gamma}\right)\right)_{\lambda}$. Since $F$ is not assumed to be a convergent power series, we actually prove more than closure under restricted analytic functions by showing that $F\left(\varepsilon_{1}, \ldots, \varepsilon_{n}\right) \in \mathbb{R}\left(\left(\tau^{\Gamma}\right)\right)_{\lambda}$. Put

$$
S:=\operatorname{supp}\left(\varepsilon_{1}\right) \cup \ldots \cup \operatorname{supp}\left(\varepsilon_{n}\right),
$$

so $S \subseteq \Gamma^{>0}$ is well ordered of order type $\sigma<\lambda$. Since $\operatorname{supp}\left(F\left(\varepsilon_{1}, \ldots, \varepsilon_{n}\right)\right)$ $\subseteq[S]$, the last lemma implies that $\operatorname{supp}\left(F\left(\varepsilon_{1}, \ldots, \varepsilon_{n}\right)\right)$ has order type at most $\omega^{\sigma}<\lambda$.

REMARK. Among the $\varepsilon$-numbers are all uncountable cardinals, where as usual we identify the cardinal $\aleph_{\alpha}$ with the initial ordinal $\omega_{\alpha}$.

These lemmas lead to a revealing picture of $\operatorname{No}(\lambda)$ for $\varepsilon$-numbers $\lambda$.

Proposition 4.7. Let $\lambda$ be an $\varepsilon$-number. Then

(1) $\mathbf{N o}(\lambda)=\bigcup_{\mu} \mathbb{R}\left(\left(\tau^{\mathrm{No}(\mu)}\right)\right)_{\lambda}$, where $\mu$ ranges over the additive ordinals $<\lambda$ (equivalently, $\mu$ ranges over the multiplicative ordinals $<\lambda$ ),

(2) $\operatorname{No}(\lambda)$ is a real closed subfield of $\mathbf{N o}$, and is closed under the restricted analytic functions of No,

(3) $\mathbf{N o}(\lambda)=\mathbb{R}\left(\left(\tau^{\mathbf{N o}(\lambda)}\right)\right)_{\lambda}$ if and only if $\lambda$ is a regular cardinal.

Proof. Let $a$ be a surreal number with Conway name $\sum_{\alpha<\beta} \omega^{y_{\alpha}} \cdot r_{\alpha}$. By Lemmas 4.2 and 4.1 we have $\beta \leq l(a)$ and $l\left(y_{\alpha}\right) \leq l\left(\omega^{y_{\alpha}}\right) \leq l(a)$ for all $\alpha<\beta$. Thus if $a \in \mathbf{N o}(\lambda)$, then $\beta<\lambda$ and there is an additive (even multiplicative) $\mu<\lambda$ with $y_{\alpha} \in \mathbf{N o}(\mu)$ for all $\alpha<\beta$. Conversely, if $\beta<\lambda$, and there exists an additive ordinal $\mu<\lambda$ such that all $y_{\alpha}$ belong to $\mathbf{N o}(\mu)$, then $a \in \operatorname{No}(\lambda)$ by Lemma 4.2(2). This proves (1). Then (2) follows from Lemma 4.6. As to (3), suppose $\lambda$ is not a regular cardinal. This means we can take a strictly increasing sequence $\left(\mu_{\alpha}\right)_{\alpha<\beta}$ of ordinals $<\lambda$ that is cofinal in $\lambda$ with $\beta<\lambda$. Then $\sum_{\alpha<\beta} \omega^{-\mu_{\alpha}} \in \mathbb{R}\left(\left(\tau^{\mathbf{N o}(\lambda)}\right)\right)_{\lambda} \backslash \mathbf{N o}(\lambda)$ by part (1). Suppose next that $\lambda$ is a regular cardinal, and let $a \in \mathbb{R}\left(\left(\tau^{\mathrm{No}(\lambda)}\right)\right)_{\lambda}$. It remains to show that then $a \in \mathbf{N o}(\lambda)$. Let $a$ have Conway name $\sum_{\alpha<\beta} \omega^{y_{\alpha}} \cdot r_{\alpha}$. Then $\beta<\lambda$, hence the subset $\left\{l\left(y_{\alpha}\right): \alpha<\beta\right\}$ of $\lambda$ is not cofinal in $\lambda$, and therefore $a \in \mathbb{R}\left(\left(\tau^{\mathbf{N o}(\mu)}\right)\right)_{\lambda}$, for some additive $\mu<\lambda$.

REMARKS. Let $\lambda$ be an $\varepsilon$-number.

(1) Here is an alternative phrasing of part (1) of the proposition: $A$ surreal number a with Conway name $\sum_{\alpha<\beta} \omega^{y_{\alpha}} \cdot r_{\alpha}$ belongs to $\mathbf{N o}(\lambda)$ if and only if $\beta<\lambda$ and there is an ordinal $\mu<\lambda$ such that $l\left(y_{\alpha}\right)<\mu$ for all $\alpha<\beta$.

(2) By Lemma 4.1 we deduce that for all surreal $y, y \in \operatorname{No}(\lambda)$ if and only if $\omega^{y} \in \mathbf{N o}(\lambda)$. 
(3) By part (1) of the proposition $\mathbf{N o}(\lambda)$ is a subfield of $\mathbb{R}\left(\left(\tau^{\mathbf{N o}(\lambda)}\right)\right)$, and has the latter as maximal immediate extension with respect to the natural valuation on both fields. (The natural valuation on an ordered field extension of $\mathbb{R}$ has as its valuation ring the convex hull of $\mathbb{R}$ in that field.) However, $\mathbf{N o}(\lambda)$ is not dense in $\mathbb{R}\left(\left(\tau^{\mathbf{N o}(\lambda)}\right)\right)$ : the element $\sum_{0<\alpha<\lambda} \omega^{1 / \alpha}$ of $\mathbb{R}\left(\left(\tau^{\mathbf{N o}(\lambda)}\right)\right)$ is clearly at distance $>1$ from every element of $\mathbf{N o}(\lambda)$.

We now proceed to a converse of the proposition.

Lemma 4.8. Suppose $\alpha>1$ is not an $\varepsilon$-number. Then $l\left(\omega^{-\alpha}\right)<\omega^{\alpha}$.

Proof. The surreal number $-\alpha$ of length $\alpha$ is a sequence consisting entirely of minuses. Hence by [6], p. 94, the surreal number $\omega^{-\alpha}$ is a sequence consisting of a plus followed by $\omega \dot{\times} \alpha$ minuses. Thus $l\left(\omega^{-\alpha}\right)=\omega \dot{\times} \alpha<\omega^{\alpha}$, where the inequality uses the fact that $\alpha$ is not an $\varepsilon$-number.

Corollary 4.9. $\mathbf{N o}(\lambda)$ is a subfield of No if and only if $\lambda$ is an $\varepsilon$ number.

Proof. Suppose $\operatorname{No}(\lambda)$ is a subfield of No. Then $\lambda=\omega^{\alpha}$ for some $\alpha>0$ by Corollary 3.1. The last lemma implies that then $\alpha$ is an $\varepsilon$-number, and thus $\lambda=\alpha$ is an $\varepsilon$-number.

The other direction is part of the last proposition.

The next two results throw further light on the structure of the fields $\operatorname{No}(\lambda)$ where $\lambda$ is an $\varepsilon$-number. The first one is also used in the next section in showing that these fields are closed under exponentiation and taking logarithms.

Lemma 4.10. Let $F\left(X_{1}, \ldots, X_{n}\right) \in \mathbb{R}\left[\left[X_{1}, \ldots, X_{n}\right]\right]$. Let $\varepsilon_{1}, \ldots, \varepsilon_{n}$ be infinitesimal surreal numbers, and let the ordinal $\mu \geq \omega$ be an upper bound on $l\left(\varepsilon_{1}\right), \ldots, l\left(\varepsilon_{n}\right)$. Then $l\left(F\left(\varepsilon_{1}, \ldots, \varepsilon_{n}\right)\right) \leq \omega^{(\omega+n) \mu}$.

Proof. Put $S:=\operatorname{supp}\left(\varepsilon_{1}\right) \cup \ldots \cup \operatorname{supp}\left(\varepsilon_{n}\right)$, so $S$ is a well ordered set of positive surreals, and by Lemma $4.2(1)$ its order type is at most $n \mu$. Hence, by Lemma 4.5, the additive monoid $[S]$ generated by $S$ in No is well ordered of order type at most $\omega^{n \mu}$. The support of $F\left(\varepsilon_{1}, \ldots, \varepsilon_{n}\right)$ is contained in $[S]$, so this support has order type at most $\omega^{n \mu}$.

Consider a term $\omega^{y} . r$ in the Conway name of $F\left(\varepsilon_{1}, \ldots, \varepsilon_{n}\right)$. Then $\omega^{y} . r$ is a finite sum of products of the form $r_{0}\left(\omega^{y_{1}} \cdot r_{1} \cdots \omega^{y_{N}} \cdot r_{N}\right)$ where $r_{0} \in \mathbb{R}$ and each factor $\omega^{y_{j}} . r_{j}$ is a term in the Conway name of some $\varepsilon_{i}$. By Lemma 4.2(2) and Proposition 3.6 such a product has length at most $\omega \mu^{N}$. Using the Cantor normal form of $\mu$ one verifies easily that $n \omega \mu^{N}<\omega^{\omega \mu}$ for all $n \in \mathbb{N}$. Hence $l\left(\omega^{y} . r\right) \leq \omega^{\omega \mu}$. Now Lemma 4.2(3) implies

$$
l\left(F\left(\varepsilon_{1}, \ldots, \varepsilon_{n}\right)\right) \leq \omega^{n \mu} \omega^{\omega \mu}=\omega^{(\omega+n) \mu} .
$$


Proposition 4.11. Let a be a non-zero surreal number. Then

$$
l\left(a^{-1}\right) \leq \omega^{\omega^{3 l(a)+3}} .
$$

If moreover $a>0$ and $d$ is a positive integer, then also

$$
l\left(a^{1 / d}\right) \leq \omega^{\omega^{3 l(a)+3}} .
$$

Proof. Write $a=r \omega^{y}(1-\varepsilon)$ with $r \in \mathbb{R} \backslash\{0\}$ and $\varepsilon$ infinitesimal. Then $\varepsilon=1-a r^{-1} \omega^{-y}$. Now $l\left(\omega^{-y}\right) \leq \omega^{l(-y)} \leq \omega^{l(a)}$ since $l(-y)=l(y) \leq l\left(\omega^{y}\right) \leq$ $l(a)$. Hence by the Remark following Corollary 4.3 we have

$$
l(\varepsilon)=l\left(1-a r^{-1} \omega^{-y}\right) \leq 1+l(a)^{2} l\left(r^{-1} \omega^{-y}\right) \leq 1+\omega^{1+3 l(a)},
$$

where we also used $l(a) \leq \omega^{l(a)}$. Let $F(X):=1+X+X^{2}+\ldots=\sum X^{n} \in$ $\mathbb{R}[[X]]$. Then by the previous lemma

$$
l(F(\varepsilon)) \leq \omega^{(\omega+1)\left(1+\omega^{1+3 l(a)}\right)} .
$$

From $a^{-1}=r^{-1} \omega^{-y} F(\varepsilon)$ we then obtain

$$
l\left(a^{-1}\right) \leq \omega \omega^{l(a)} \omega^{(\omega+1)\left(1+\omega^{1+3 l(a)}\right)} \leq \omega^{\omega^{3 l(a)+3}} .
$$

Now assume $a>0$, so $r>0$. Put $G(X):=\sum_{n=0}^{\infty}(-1)^{n}\left(\begin{array}{c}1 / d \\ n\end{array}\right) X^{n} \in \mathbb{R}[[X]]$. Then $a^{1 / d}=r^{1 / d} \omega^{y / d} G(\varepsilon)$, so

$$
\begin{aligned}
l\left(a^{1 / d}\right) & \leq l\left(r^{1 / d}\right) \omega^{l(y / d)} l(G(\varepsilon))^{2} \quad \text { (by Remark following Corollary 4.3) } \\
& \leq \omega \omega^{\omega l(y)} \omega^{(2 \omega+2)\left(1+\omega^{1+3 l(a)}\right)} \leq \omega^{\omega^{3 l(a)+3}}
\end{aligned}
$$

where we used $l(y) \leq l(a) \leq \omega^{l(a)}$.

REMARK. This double $\omega$-exponential bound for $l\left(a^{-1}\right)$ cannot be replaced by a polynomial bound like $l\left(a^{-1}\right) \leq \omega^{2} l(a)^{3}$ : for $a=\omega^{-6}$ we have $l(a)=\omega .6$ by the proof of Lemma 4.8 , so $l\left(a^{-1}\right)=\omega^{6}>\omega^{2} l(a)^{3}$. We leave open the possibility that the double $\omega$-exponential bound can be replaced by a single $\omega$-exponential bound.

5. Bounds on lengths of exponentials and logarithms. Next we come to bounds involving Gonshor's exponential function exp. We shall use the results in [6], Ch. 10, parts C and D, which relate exponentiation to $\omega$ exponentiation via the order preserving bijection $g: \mathbf{N o}^{>0} \rightarrow$ No given by

$$
\exp \left(\omega^{a}\right)=\omega^{\omega^{g(a)}} \quad \text { for } a>0 .
$$

Lemma 5.1. For surreal $a>0$ we have $l(g(a)) \leq l(a)+1$.

Proof. By [6], Th. 10.11, we have $g(a)=\left\{c, g\left(a^{\prime}\right) \mid g\left(a^{\prime \prime}\right)\right\}$ where $c$ is such that $a$ and $\omega^{c}$ have the same archimedean class, $a^{\prime}$ ranges over the positive surreals simpler and less than $a$, and $a^{\prime \prime}$ ranges over the positive surreals simpler and greater than $a$. Assume inductively that $l\left(g\left(a^{\prime}\right)\right) \leq l\left(a^{\prime}\right)+1$ and $l\left(g\left(a^{\prime \prime}\right)\right) \leq l\left(a^{\prime \prime}\right)+1$ for those $a^{\prime}$ and $a^{\prime \prime}$. Thus $l\left(g\left(a^{\prime}\right)\right)<l(a)+1$ and 
$l\left(g\left(a^{\prime \prime}\right)\right)<l(a)+1$ for those $a^{\prime}$ and $a^{\prime \prime}$. Also, $l(c) \leq l\left(\omega^{c}\right) \leq l(a)<l(a)+1$. The desired result now follows from [6], Th. 2.3.

The bound in this lemma is optimal, since $g(a)=a+1$ when $a$ is an $\varepsilon$-number; see [6], Th. 10.14.

LEMMA 5.2. For each surreal number a we have

$$
l(\exp (a)) \leq \omega^{\omega^{2 l(a)+3}} .
$$

Proof. Write $a=b+r+\varepsilon$ where $b$, the "purely infinite" part of $a$, has Conway name $b=\sum_{\alpha<\beta} \omega^{y_{\alpha}} . r_{\alpha}$ with all exponents $y_{\alpha}>0, r \in \mathbb{R}$, and infinitesimal $\varepsilon$. Let $E(X):=\sum_{n=0}^{\infty}(1 / n !) X^{n} \in \mathbb{R}[[X]]$. Then $\exp (a)=$ $\omega^{c} e^{r} E(\varepsilon)$ where $c=\sum_{\alpha<\beta} \omega^{g\left(y_{\alpha}\right)} \cdot r_{\alpha}$ according to [6], Th. 10.13. First we determine a bound on $l(c)$. Using the last lemma we have

$$
l\left(\omega^{g\left(y_{\alpha}\right)} \cdot r_{\alpha}\right) \leq \omega \omega^{l\left(g\left(y_{\alpha}\right)\right)} \leq \omega^{l\left(y_{\alpha}\right)+2} .
$$

An earlier lemma gives

$$
l\left(y_{\alpha}\right) \leq l\left(\omega^{y_{\alpha}}\right) \leq l\left(\omega^{y_{\alpha}} \cdot r_{\alpha}\right) \leq l(a) .
$$

As also $\beta \leq l(a) \leq \omega^{l(a)}$, we obtain

$$
l(c) \leq \beta \omega^{l\left(y_{\alpha}\right)+2} \leq l(a) \omega^{l(a)+2} \leq \omega^{2 l(a)+2} .
$$

Since $\varepsilon=a-(b+r)$ and $b+r \leq_{s} a$ by [4], Th. 15(i), we have $l(\varepsilon) \leq 2 l(a)$, so that by Lemma 4.10 ,

$$
l(E(\varepsilon)) \leq \omega^{(\omega+1) 2 l(a)} \leq \omega^{\omega^{l(a)+2}}
$$

and thus by the Remark following Corollary 4.3,

$$
l(\exp (a)) \leq l\left(\omega^{c}\right) l\left(e^{r}\right) l(E(\varepsilon))^{2} \leq \omega^{\omega^{2 l(a)+2}+1+2 \omega^{l(a)+2}} \leq \omega^{\omega^{2 l(a)+3}} .
$$

Let $\log$ denote the inverse of exp. There is an inductive definition of $\ln (x)$ for surreal $x$ of the form $\omega^{y}$ in [6], p. 161, and [6], Th. 10.8 shows that $\log (x)=\ln (x)$ for such $x$.

LEMMA 5.3. For each surreal number y we have $l\left(\log \left(\omega^{y}\right)\right) \leq \omega^{4 \omega l(y)^{2}}$.

Proof. This is clearly true for $y=0$. Since $\log (\omega)=\omega^{1 / \omega}$ (see [6], p. 161), the desired inequality follows for $y=1$ by using $l(1 / \omega)=\omega$ and Lemma 4.1. For $y=-1$ the inequality follows from the case $y=1$ since $\log \left(\omega^{-1}\right)=-\log \left(\omega^{1}\right)$. Suppose next that $l(y)>1$ and that the lemma holds for smaller values of $l(y)$. Then the desired inequality follows from the inductive definition of $\ln \left(\omega^{y}\right)$ in [6], p. 161, in combination with Lemma 4.1, the remark following Corollary 4.3, and [6], Th. 2.3.

LEMma 5.4. For each surreal number $a>0$ we have

$$
l(\log (a)) \leq \omega^{\omega^{3 l(a)+3}} .
$$


Proof. Write $a=r \omega^{y}(1-\varepsilon)$ with $0<r \in \mathbb{R}$ and $\varepsilon$ infinitesimal. Let $H(X):=-\sum_{n=1}^{\infty}(1 / n) X^{n} \in \mathbb{R}[[X]]$. Then $\log (a)=\log (r)+\log \left(\omega^{y}\right)+H(\varepsilon)$. As in the proof of Proposition 4.11 we have

$$
l(H(\varepsilon)) \leq \omega^{(\omega+1)\left(1+\omega^{1+3 l(a)}\right)} .
$$

The desired inequality now follows easily from the last lemma.

The bounds in this section, together with the results in [3] and Proposition 4.7, imply the following.

Corollary 5.5. Let $\lambda$ be an $\varepsilon$-number. Then the field $\operatorname{No}(\lambda)$ is closed under exponentiation, and under taking logarithms of positive elements. The field $\operatorname{No}(\lambda)$ equipped with the restricted analytic functions and exponentiation induced by $\mathbf{N o}$ is an elementary substructure of ( $\mathbf{N o}_{\text {an }}$, $\left.\exp \right)$ and an elementary extension of $\left(\mathbb{R}_{\mathrm{an}}, e^{x}\right)$.

6. Some other initial subfields of No. Let $\Gamma$ be an initial additive subgroup of No. (Recall that "initial" means that if $y \in \Gamma$ and $x<_{s} y$, then $x \in \Gamma$. In particular, $\mathbf{N o}(\lambda)$ is an initial additive subgroup of No for additive $\lambda$.) As in [4] we let $\operatorname{On}(\Gamma)$ denote the set of ordinals in $\Gamma$. Note that $O n(\Gamma)$ is cofinal in $\Gamma$, and is itself an (additive) ordinal; in fact, it is the smallest ordinal $>\Gamma$.

These properties carry over to $\mathbb{R}\left(\left(\tau^{\Gamma}\right)\right)$ as follows.

Proposition 6.1. $\mathbb{R}\left(\left(\tau^{\Gamma}\right)\right)$ is an initial subfield of No, and On $\left(\mathbb{R}\left(\left(\tau^{\Gamma}\right)\right)\right)$ $=\omega^{O n(\Gamma)}$.

Proof. The proof of [4], Th. 18, shows that $\mathbb{R}\left(\left(\tau^{\Gamma}\right)\right)$ is an initial subfield of No. The elements $\omega^{y}$ with $y \in \Gamma$ are cofinal in $\mathbb{R}\left(\left(\tau^{\Gamma}\right)\right)$, and hence the ordinals $\omega^{\alpha}$ with $\alpha \in O n(\Gamma)$ are cofinal in $\mathbb{R}\left(\left(\tau^{\Gamma}\right)\right)$. Since $O n(\Gamma)$ is additive it follows that $\operatorname{On}\left(\mathbb{R}\left(\left(\tau^{\Gamma}\right)\right)\right)=\omega^{O n(\Gamma)}$.

In particular, if $\lambda$ is an $\varepsilon$-number, then $\mathbb{R}\left(\left(\tau^{\mathrm{No}(\lambda)}\right)\right)$ is an initial subfield of No, and the ordinals in it are exactly the ordinals $<\lambda$.

\section{References}

[1] J. Conway, On Numbers and Games, Academic Press, 1976.

[2] L. van den Dries, Tame Topology and o-Minimal Structures, London Math. Soc. Lecture Note Ser. 248, Cambridge Univ. Press, 1998.

[3] L. van den Dries, A. Macintyre and D. Marker, The elementary theory of restricted analytic fields with exponentiation, Ann. of Math. 140 (1994), 183-205.

[4] P. Ehrlich, Number systems with simplicity hierarchies: a generalization of Conway's theory of surreal numbers, J. Symbolic Logic, to appear.

[5] -, Absolutely saturated models, Fund. Math. 133 (1989), 41-46. 
[6] H. Gonshor, An Introduction to the Theory of Surreal Numbers, London Math. Soc. Lecture Note Ser. 110, Cambridge Univ. Press, 1986.

[7] F. Hausdorff, Set Theory, Chelsea, New York, 1957.

[8] J. Lurie, Review of [1], J. Symbolic Logic 63 (1998), 1602-1604.

[9] A. Macintyre and A. Wilkie, On the decidability of the real exponential field, in: Kreiseliana, P. Odifreddi (ed.), A. K. Peters, Wellesley, MA, 1996, 441-467.

[10] A. Wilkie, Model completeness results for expansions of the ordered field of real numbers by restricted Pfaffian functions and the exponential function, J. Amer. Math. Soc. 9 (1996), 1051-1094.

Department of Mathematics

University of Illinois at Urbana-Champaign

Urbana, IL 61801, U.S.A.

E-mail: vddries@math.uiuc.edu
Department of Philosophy

Ohio University

Athens, OH 45701, U.S.A.

E-mail: ehrlich@ohiou.edu

Received 6 May 2000;

in revised form 29 August 2000 